\title{
INEQUALITIES FOR EIGENVALUES OF COMPACTLY PERTURBED UNITARY OPERATORS
}

\author{
MiCHAEL GiL'
}

Abstract. We consider the operator $A=U+K$, where $U$ is a unitary operator and $K$ is a compact one. An eigenvalue $\lambda$ of $A$ is said to be a non-unitary one, if $|\lambda| \neq 1$. We derive inequalities for sums of absolute values of the non-unitary eigenvalues. Applications of these inequalities to operator functions, spectrum perturbations and operator equations are also discussed.

Mathematics subject classification (2010): 47B10, 47A55, 47B10, 47A62.

Keywords and phrases: Hilbert space, eigenvalues, inequalities Schatten - von Neumann operators, resolvent, operator functions, spectrum perturbations, Sylvester operator equations.

\section{REFERENCES}

[1] A. A. BEN, Fixed points, eigenvalues and surjectivity for (ws)-compact operators on unbounded convex sets, Cent. Eur. J. Math. 11, (2013), no. 1, 85-93.

[2] A. A. BONDAR, AND YA. M. DYMARSKII, Submanifolds of compact operators with fixed multiplicities of eigenvalues, Ukrainian Math. J. 63, (2012), no. 9, 1349-1360.

[3] Yu. L. DAleckit And M. G. KReIn, Stability of Solutions of Differential Equations in Banach Space, Amer. Math. Soc., Providence, R. I, 1971.

[4] M. Demuth, M. Hansmann, And G. Katriel, On the discrete spectrum of non-selfadjoint operators, J. Funct. Anal. 257 (9), (2009) 2742-2759.

[5] M. Demuth, M. Hansmann, And G. KATRIEl, Eigenvalues of non-selfadjoint operators: a comparison of two approaches, in Mathematical physics, spectral theory and stochastic analysis, vol. 232 of Oper. Theory Adv. Appl., pages 107-163, Birkhäuser/Springer, Basel, 2013.

[6] M. Demuth, F. Hanauska, M. Hansmann, And G. Katriel, Estimating the number of eigenvalues of linear operators on Banach spaces, J. Funct. Anal. 268 (4), (2015) 1032-1052.

[7] M. I. GIL', Operator Functions and Localization of Spectra, Lecture Notes in Mathematics, vol. 1830, Springer-Verlag, Berlin, 2003.

[8] M. I. GIL', Lower bounds for eigenvalues of Schatten-von Neumann operators, J. of Inequalities in Pure and Appl. Mathem 8, no. 3, Article 66 (2007), 7 pp.

[9] M. I. GIL', Bounds for eigenvalues of Schatten-von Neumann operators via self-commutators, Journal of Functional Analysis 267, (2014) no. 9, 3500-3506.

[10] M. I. GIL', Inequalities for Eigenvalues of Compact Operators in a Hilbert Space, Communications in Contemporary Mathematics, online from 13 March, 2015.

[11] I. C. Gohberg And M. G. KreIn, Introduction to the Theory of Linear Nonselfadjoint Operators, Trans. Mathem. Monographs, vol. 18, Amer. Math. Soc., Providence, R. I., 1969.

[12] M. Hansmann, Variation of discrete spectra for non-selfadjoint perturbations of selfadjoint operators, Integral Equations and Operator Theory 76 (2), (2013) 163-178.

[13] M. Hansmann, Perturbation determinants in Banach spaces with an application to eigenvalue estimates for perturbed operators, Mathematische Nachrichten, online from July, 29, 2015.

[14] T. KATo, Perturbation Theory for Linear Operators, Springer-Verlag, Berlin, 1980.

[15] H. KÖNIG, Eigenvalue distribution of compact operators, vol. 16 of Operator Theory: Advances and Applications, Birkhäuser Verlag, Basel, 1986.

[16] H. MAJIDIAN AND E. BABOLIAN, An interpolation degenerate kernel method for eigenvalue problems of a class of non-compact operators, Appl. Math. Lett. 23 (2010), no. 8, 825-830. 
[17] A. G. Mazko, Matrix Equations, Spectral Problems and Stability of Dynamic Systems, Stability, Oscillations and Optimization of Systems, Scientific Publishers, Cambridge, 2008.

[18] A. PietsCh, Eigenvalues and s-Numbers, Cambridge Univesity Press, Cambridge, 1987.

[19] S. ShKARIN, Compact operators without extended eigenvalues, J. Math. Anal. Appl. 332 (2007), no. $1,455-462$. 